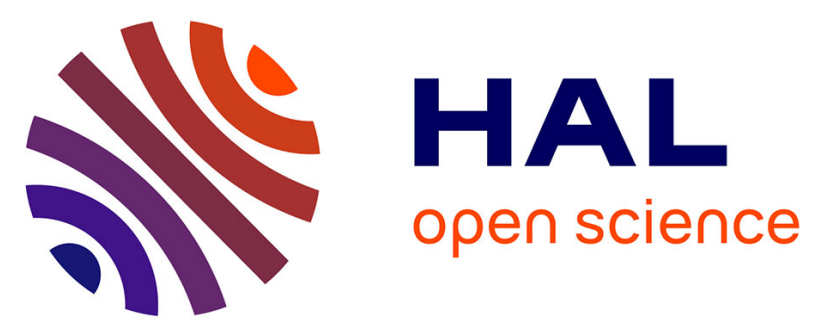

\title{
Comparative analysis of the Leishmania infantum-specific antibody repertoires and the autoantibody repertoires between asymptomatic and symptomatic dogs
}

Azza Chaabouni, Ramzi Boubaker Elandoulsi, Moez Mhadhbi, Mohamed

Gharbi, Atfa Sassi

\section{To cite this version:}

Azza Chaabouni, Ramzi Boubaker Elandoulsi, Moez Mhadhbi, Mohamed Gharbi, Atfa Sassi. Comparative analysis of the Leishmania infantum-specific antibody repertoires and the autoantibody repertoires between asymptomatic and symptomatic dogs. Veterinary Parasitology, 2018, 261, pp.9-17. 10.1016/j.vetpar.2018.07.011 . pasteur-02010342

\section{HAL Id: pasteur-02010342 \\ https://hal-riip.archives-ouvertes.fr/pasteur-02010342}

Submitted on 7 Feb 2019

HAL is a multi-disciplinary open access archive for the deposit and dissemination of scientific research documents, whether they are published or not. The documents may come from teaching and research institutions in France or abroad, or from public or private research centers.
L'archive ouverte pluridisciplinaire HAL, est destinée au dépôt et à la diffusion de documents scientifiques de niveau recherche, publiés ou non, émanant des établissements d'enseignement et de recherche français ou étrangers, des laboratoires publics ou privés. 


\section{Accepted Manuscript}

Title: Comparative analysis of the Leishmania

infantum-specific antibody repertoires and the autoantibody

repertoires between asymptomatic and diseased dogs

Authors: Azza Chaabouni, Ramzi Boubaker Elandoulsi, Moez

Mhadhbi, Mohamed Gharbi, Atfa Sassi

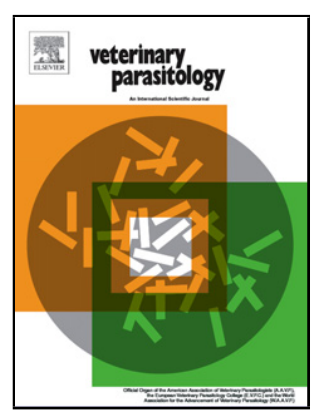

PII:

S0304-4017(18)30280-2

DOI:

https://doi.org/10.1016/j.vetpar.2018.07.011

Reference:

VETPAR 8723

To appear in: $\quad$ Veterinary Parasitology

Received date: $\quad 6-5-2018$

Revised date: $\quad 3-7-2018$

Accepted date: $\quad$ 21-7-2018

Please cite this article as: Chaabouni A, Boubaker Elandoulsi R, Mhadhbi M, Gharbi M, Sassi A, Comparative analysis of the Leishmania infantum-specific antibody repertoires and the autoantibody repertoires between asymptomatic and diseased dogs, Veterinary Parasitology (2018), https://doi.org/10.1016/j.vetpar.2018.07.011

This is a PDF file of an unedited manuscript that has been accepted for publication. As a service to our customers we are providing this early version of the manuscript. The manuscript will undergo copyediting, typesetting, and review of the resulting proof before it is published in its final form. Please note that during the production process errors may be discovered which could affect the content, and all legal disclaimers that apply to the journal pertain. 
Title: Comparative analysis of the Leishmania infantum-specific antibody repertoires and the autoantibody repertoires between asymptomatic and diseased dogs

Authors: Azza Chaabouni ${ }^{\mathrm{a}}$, Ramzi Boubaker Elandoulsi ${ }^{\mathrm{b}}$, Moez Mhadhbi ${ }^{\mathrm{c}}$, Mohamed Gharbi ${ }^{\mathrm{c}}$ and Atfa Sassi ${ }^{\mathrm{a}}$

${ }^{\text {a }}$ Laboratory of BioInformatic, BioMathematic and BioStatistics. LR16 IPT09. Institut Pasteur de Tunis, Université de Tunis El Manar, 1002. Tunisia

${ }^{\mathrm{b}}$ Lab Biotechnology and Bio-Geo Resources Valorization (LR11ES31). Higher institute of Biotechnology. BiotechPôle, BP66. 2020. Sidi Thabet. University La Manouba. Tunisia

${ }^{\mathrm{c}}$ Laboratoire de Parasitologie, École Nationale de Médecine Vétérinaire, University La Manouba, 2020. Sidi Thabet, Tunisia

\section{*Corresponding author}

Atfa Sassi. Ph.D.

Laboratory of BioInformatics, BioMathematics and BioStatistics. LR16 IPT09. Institut Pasteur of Tunis. 13, Place Pasteur. B.P. 74. Tunis-Belvédère. 1002. Tunisia.

Email: $\underline{\text { atfa.sassi@pasteur.rns.tn and atfasassi@yahoo.com }}$

Tel: + (216) 71-840-716 Ext 511

Fax: + (216) 71-791-833 


\section{Highlights}

- Dogs with cVL demonstrate higher titers of specific Tot IgG, IgG1 and IgG2.

- The production of autoantibodies starts since the subclinical phase of dogs' infection.

- Autoreactive IgG1 dominates the autoantibody repertoire of asymptomatic dogs.

- Cross-reactive IgG1 antibodies dominate the autoantibody repertoire of sick dogs

- Anti-transferrin autoantibodies are produced during both stages of dog's infection.

\section{ABSTRACT}

Leishmania (L.) infantum-infected dogs may present with a large range of clinical signs, from apparently healthy with no or few (asymptomatic dogs, AD) to several clinical signs characteristic of active infection (symptomatic dogs, SD). The present study is justified by the conflicting reports describing that either L. infantum-specific IgG1 or IgG2 antibodies may be used as isotype marker of the asymptomatic infection status and by the lack, to our knowledge, of previous analysis of the IgG sub-classes autoantibody repertoires of Leishmania-infected dogs. On the basis of clinical evaluation and laboratory testing (IFAT, parasitological examination of Giemsa-stained lymph node smears, L. infantum antigens-ELISA of total (Tot) $\mathrm{IgG}$ ), $131 \mathrm{dogs}$ were categorized as AD, SD, healthy non infected dogs (HND) from surrounding areas, and as negative control dogs (CTD). ELISA based on leishmanial native antigens or recombinant LACK and LeIF proteins showed that SD produce higher levels of specific Tot IgG, IgG1 and IgG2 antibodies than AD, and that for both clinical stages, the antibody titers of IgG2 isotype were constantly higher than those of the IgG1. The seroprevalences of Tot $\mathrm{IgG}, \mathrm{IgG} 2$ did not differ between AD and SD groups (97 and $97 \%$ in $\mathrm{SD} ; 100$ and $96 \%$ in $\mathrm{AD}$, respectively) whereas that of IgG1 was slightly lower in SD (88\% of AD versus $82 \%$ of SD). The autoantibody repertoires were analyzed by ELISA using HEp-2 extracts, ds-DNA, human albumin and transferrin as self-antigens and by Western blot using HEp-2 proteins. 
ELISA results' indicated that $\mathrm{AD}$ develop higher levels of IgG1 autoantibodies, and higher seroprevalence (50\% and $26 \%$ in $\mathrm{AD}$ and SD respectively), contrasting with lower levels and seroprevalences of Tot IgG and IgG2 (43 and 68\% for AD; 100 and 74\% for SD). Interestingly, SD showed a stronger IgG1 and particularly IgG2 reactivity with transferrin, an iron-binding protein, than $\mathrm{AD}$ and HND. Western blotting experiments produced heterogeneous IgG1 and IgG2 interand intra-groups reactivity profiles towards HEp-2 proteins, to identify a specific antigenic profile. Generated data from competitive HEp-2-ELISA using leishmanial antigens as inhibitors were in favor that IgG1 antibodies are predominantly autoantibodies to self-antigens in AD whereas they are mainly cross-reactive (Leishmania/self-antigens) autoantibodies in SD.

Key words: Leishmania infantum; Canine visceral leishmaniasis; IgG1 and IgG2 isotypes; Autoantibodies; Human transferrin. 


\section{Introduction}

Domestic dogs are the primary reservoir hosts for zoonotic visceral leishmaniasis (VL), the most widespread entity of leishmaniasis caused by the protozoa Leishmania (L.) infantum. Due to the close proximity between dogs and humans, canine cases often precede the occurrence of human cases. The disease is endemic in the Mediterranean basin, Middle East, Central Asia and Latin America (Alvar et al., 2012). Previous investigations have categorized L. infantum-infected dogs into three clinical forms: (i) asymptomatic dogs (AD) with no suggestive signs of VL or other diseases; (ii) oligosymptomatic with a maximum of three clinical signs and (iii) symptomatic dogs (SD) with characteristic clinical signs of VL (Quinnel et al., 2003; Koutinas and Koutinas, 2014). The major signs of canine VL (cVL) include hepatosplenomegaly, lymphadenopathy, cutaneous lesions, keratoconjunctivitis, opaque bristles, alopecia, apathy, onychogriphosis, anorexia, anaemia and severe weight loss (Bettini and Gradoni, 1986; Abbehusen et al., 2017). The serological diagnosis of L. infantum infection in AD, potential carriers of Leishmania parasite, remains so far problematic and their living near human hosts constitute a serious issue for public health.

Laboratory findings showed that $\mathrm{cVL}$ is characterized by the suppression of the cellular immune responses to specific antigens and as the disease progresses to mitogens, as well as a strong up-regulation of specific and "non specific" humoral responses (Pinelli et al., 1994; Cabral et al., 1998). The main hypothesis that were generally retained to explain hypergammaglobulinemia induction and autoantibodies appearance are that (i) parasite-derived antigens may activate $\mathrm{T}$ helper (Th) 2 lymphocytes for the production of B-cell stimulating interleukins (IL) such as IL-4, IL-6 and IL-10; (ii) parasite antigens may directly act as mitogenic substances to B cells; (iii) Leishmania parasite induces by cell damage and tissue destruction the release of host's autoantigens which in turn causes autoreactivity; (iv) molecular mimicry between Leishmania antigens and host's selfantigens yields also to B cells activation. Polyclonal activation of B cells results in an increase of the levels of non specific antibodies including natural antibodies, the concentration of circulating immune complexes and to a lesser extent, the levels of Leishmania-specific antibodies (Greenwood, 
1974; Pateraki et al., 1983; Galvão-Castro et al., 1984; Böhme et al., 1986; Argov et al., 1989; Louzir et al., 1994; Liberopoulos et al., 2003; Montes et al., 2007; Tunccan et al, 2012; Jamal et al., 2017).

Numerous investigations of human, mouse and dog models have attempted to determine the significance and prognostic value of the Leishmania-specific IgG subclasses in parasite-infected hosts (Anam et al., 1999; Bhattacharyya et al., 2014; Sassi et al., 2015; Day et al., 2007). Indeed, the levels of specific IgG1 and IgG2 antibodies directed towards crude, soluble promastigote or amastigote lysates, were extensively investigated by enzyme-linked immunosorbent assay (ELISA) and/or by western blotting (WB), in cases of symptomatic (SD) and asymptomatic dogs (AD) that have been naturally or experimentally infected with L. infantum. Conflicting results were reported, and it has been suggested that the lack of correlation between IgG subclasses and parasite infection stage could be due to a weak sensitivity of the used leishmanial antigens and/or of the anti-IgG subclasses commercial antibodies, i.e. polyclonal versus monoclonal antibodies (Quinnell et al., 2003; Day et al., 2007; Carson et al., 2010). Some studies associated asymptomatic infection with higher IgG2 levels compared to IgG1 (Deplazes et al., 1995; Iniesta et al., 2005), while others observed the opposite during active clinical infections (Bourdoiseau et al., 1997; Nieto et al., 1999; Boceta et al., 2000; Almeida et al., 2005; Reis et al., 2006; Cardoso et al., 2007; Teixeira Neto et al., 2010). Carson et al. (2010) described that the levels of both IgG subclasses are higher in SD whereas in a more recent study Lima et al. (2017) reported higher IgG1 levels for SD but no differences in those of IgG2 between AD and SD.

It is in this context we aimed the present study to (i) analyze the profile of specific IgG1 and IgG2 antibodies in a cohort of domestic dogs living in Tunisian endemic areas for L. infantum transmission, in order to check whether IgG1 or IgG2 may characterize dog's clinical status and (ii) investigate whether the production of autoantibodies played a role in the conflicting data reported in the literature. 


\section{Materials and methods}

\subsection{Animals and sera}

The Ethical Committee of the Institute Pasteur of Tunis provided its approval for the study protocol. A total number of 131 dogs' sera were collected after the owners' consent. Sera were stored at $-20^{\circ} \mathrm{C}$ until analyzed. Based on clinical examination, parasitological and serological analyses, three dog groups were constituted.

The first group consisted of 34 naturally infected sick dogs, none have received specific anti-Leishmania drugs. Dogs, originated from Bizerte, Tunis, Ariana, Manouba and Ben Arous districts, were presented to the Parasitology Department at the National School of Veterinary Medicine of Sidi Thabet (Tunis. Tunisia) between September 2009 and December 2011 for diagnostic purposes. Dogs were submitted to clinical examination and symptoms such as apathy, weight lost, skin lesions, lymph node and spleen enlargement, onychogryphosis and anemia, were recorded. For each dog, a score varying between zero (no sign) to three (severe) was attributed depending on the symptom intensity and a total clinical score (TCS) was attributed (Quinnell et al., 2003). The TCS ranged from 3 to 16 (mean 11.2 +3.6). A direct detection of Leishmania parasite, performed by the examination of Giemsa-stained lymph node aspirates, showed nine out of thirtyfour $(26 \%)$ negative dogs. All serum samples tested positive by the immunofluorescence antibody test (IFAT) (serum dilution > 1/80).

The second group consisted of 71 serum samples collected from healthy dogs during epidemiological investigations of Tunisian L. infantum endemic foci. Physical examination did not show any clinical symptoms that could be attributed to VL or any other diseases. This group was subdivided into two groups based on the reactivity of their Tot IgG towards L. infantum antigens: a group of healthy non-infected dogs (HND) with IgG reactivity lower than the cut-off value and a second group of asymptomatically infected dogs (AD) with IgG reactivity higher than the cut-off value. 
The third group consisted of 26 archived negative serum samples obtained from healthy control dogs (CTD) with laboratory negative results. This group was used for the determination of ELISA cut-off values.

\subsection{Antigens for immunoenzymatic assays}

L. infantum (MHOM/TN/80/IPT-1) promastigotes were maintained in RPMI-1640 medium supplemented with $2 \mathrm{mM}$ L-glutamine, 100 units/ml Penicillin, $100 \mu \mathrm{g} / \mathrm{ml}$ Streptomycin and 5\% of heat-inactivated fetal bovine serum (FBS) at $26^{\circ} \mathrm{C}$. Parasites were collected at the stationary phase, washed three times in cold phosphate buffered saline (PBS) $\mathrm{pH} 7.2$ by centrifugation at 3,000 $\mathrm{g}$ for 15 min and then lysed in $10 \mathrm{mM}$ Tris $1 \mathrm{mM}$ EDTA (pH 8.0) PMSF $1 \mathrm{mM}$ by six cycles of freezing at $-80^{\circ} \mathrm{C}$ and thawing at room temperature (RT). Preparations were centrifuged at $12,500 \mathrm{rpm}$ for 45 minutes at $+4^{\circ} \mathrm{C}$ and the supernatant was harvested and stored at $-70^{\circ} \mathrm{C}$ until use in ELISA experiments. Recombinant proteins, Leishmania homologue of receptors for activated C kinase (LACK) and Leishmania elongation initiation factor (LeIF), were kindly provided by C. Bahloul (Institut Pasteur de Tunis. Tunisia). Human albumin and human transferrin were purchased from Sigma (France).

Human epithelial (HEp-2) adherent cells were cultured in RPMI-1640 medium supplemented with $5 \% \mathrm{FBS}$, at $37^{\circ} \mathrm{C}$ and $5 \% \mathrm{CO}_{2}$ in a humidified incubator. Cells were grown to 5 x $10^{5}$ cells/ $\mathrm{ml}$ then harvested by centrifugation for $5 \mathrm{~min}$ at $2000 \mathrm{rpm}$. Cells' pellets were washed three times with PBS and lysed on ice for $30 \mathrm{~min}$ in Tris- $\mathrm{HCl} 50 \mathrm{mM} \mathrm{pH} 8$ containing $0.1 \%$ Triton X100. Supernatant was collected after centrifugation at 12,000 rpm for $30 \mathrm{~min}$ and used in ELISA for the detection of autoantibodies.

Protein concentrations of leishmanial antigens and HEp-2 preparations were determined by the BCA kit (Sigma) based on a bovine serum albumin standard curve.

\subsection{Assessment of the Leishmania-specific antibody responses by ELISA}


Ninety-six well microplates (MaxiSorp. Nunc) were coated with 100 $\mu 1 /$ well of leishmanial soluble antigens $(5 \mu \mathrm{g} / \mathrm{ml})$, LACK or LeIF recombinant proteins $(2 \mu \mathrm{g} / \mathrm{ml})$ diluted in bicarbonate buffer $(0.1 \mathrm{M} \mathrm{NaH} \mathrm{CO} 3, \mathrm{pH} 8.1)$. After overnight incubation at $4{ }^{\circ} \mathrm{C}$, wells were washed twice with PBS containing $0.05 \%$ Tween-20 (PBS-Tw) and received 200 $\mu 1$ of $2 \%$ skimmed milk (PBS-Twmilk) for $1 \mathrm{H}$ at $37^{\circ} \mathrm{C}$ to reduce non-specific binding. Blocking buffer was removed by two washes and dog's sera, diluted in PBS- Tw- milk as described in figure legends, were added to wells. Plates were incubated $1 \mathrm{H}$ at $37{ }^{\circ} \mathrm{C}$ then washed seven times. One hundred microliters of polyclonal peroxidase-conjugated anti-dog antibodies, diluted in PBS-Tw-milk, was added and incubated for $1 \mathrm{H}$ at RT. The plates were washed six times and the substrate ortho-phenylenediamine $(0.4 \mathrm{mg} / \mathrm{ml})$ dissolved in $100 \mathrm{mM}$ citrate buffer pH 5.0 containing $0.03 \%$ hydrogen peroxide was added. Reactions were stopped by the addition of $50 \mu \mathrm{l} /$ well of $3 \mathrm{~N}$ sulfuric acid and optical densities (OD) were recorded at $492 \mathrm{~nm}$ using an ELISA reader. The conjugates anti-dog IgG antibodies (Sigma), anti-IgG1, and anti-IgG2 (both from Bethyl Laboratories) were used at a dilution of 1:10,000, $1: 2,000$ and 1:20,000, respectively.

\subsection{Assessment of the serum autoantibody levels by ELISA}

The detection of IgG, IgG1 and IgG2 autoantibodies in dog's sera was performed by ELISA as described above using microtitre plates coated with HEp-2 extracts (Binder et al., 2005), with human albumin or human transferrin $(5 \mu \mathrm{g} / \mathrm{ml})$ or with double-stranded deoxyribonucleic acid (dsDNA, $10 \mu \mathrm{g} / \mathrm{ml}$ ), diluted in bicarbonate buffer. Dogs' sera were tested at dilutions given in figures' legends.

\subsection{Analysis of the specificity of IgG1 and IgG2 autoantibodies by competitive ELISA}

The specificity of the IgG1 or IgG2 binding to leishmanial antigens was examined by competitive ELISA. Dogs' serum samples obtained from 8 AD and 7 SD positive for reactivity with HEp-2 antigens were diluted at 1:150, 1:300, 1:600 and 1:1,200 then pre-incubated (v/v) for 3 to 4 
$\mathrm{H}$ at $37^{\circ} \mathrm{C}$ with (W) $100 \mu \mathrm{g} / \mathrm{ml}$ of leishmanial antigens or without (WO, buffer only). The reactivity of IgG1 and IgG2 antibodies was measured, as described above, by ELISA on HEp-2 coated plates. Results were expressed as the mean OD \pm 1 SEM of the antibody binding with or without inhibitor. Percentage of inhibition was determined as follows: 100-[OD w/OD ${ }_{\text {WO }}$ 100].

\subsection{Western blotting}

Proteins from HEp-2 lysates were separated by 15\% SDS-PAGE under reducing conditions and transferred to nitrocellulose membranes (GE Healthcare, Buckinghamshire, UK). Nitrocellulose strips were placed in PBS, 0.1\% Tween-20 (PBS-Tw), 2\% skim milk and incubated overnight at $4^{\circ} \mathrm{C}$. Incubation of nitrocellulose strips with dog sera, diluted in PBS-Tw-2\% milk, was carried out during $1 \mathrm{H}$ at RT with mechanical agitation. Goat anti-dog $\operatorname{IgG} 1(1 / 3,000)$ and sheep anti-dog $\operatorname{IgG} 2$ $(1 / 25,000)$ both conjugated to HRP were incubated for 45 and $30 \mathrm{~min}$ at RT, respectively. Unbound antibodies were removed by extensive washing with PBS-Tw. Peroxidase enzymatic reactions were detected by an enhanced chemiluminescence (ECL) assay used according to the manufacturer's instructions (GE Healthcare, Buckinghamshire, UK). The molecular weight (MW) of the more reactive bands was determined in kDa using standard proteins of known MWs.

\subsection{Statistical analysis}

Data analyses were performed using the GraphPad Prism software. Cut-off values for positivity were defined as being two or three standard deviations (SD) from the mean absorbance of the negative control serum samples (CTD). Normality of the data was established using the Kolmogorov-Smirnoff test. Differences in the OD median or mean values of antibody reactivity between groups were evaluated in the $95 \%$ confidence interval using paired or unpaired Student's $t$ test and the Mann-Whitney-U test, as indicated in figure's legends. Differences between groups were considered significant when $P$ values were $<0.05$. Statistical associations were established using the Pearson's correlation coefficient (r). 


\section{Results}

\subsection{Anti-Leishmania Tot IgG, IgG1 and IgG2 levels in dogs' sera}

The levels of Tot IgG and of two IgG subclasses to L. infantum extracts were measured for all study groups. Results, shown in Fig. 1A, indicate a stronger reactivity of Tot IgG and IgG2 towards leishmanial antigens in SD sera, comparatively to IgG1 antibodies $(P<0,000001)$. One $\operatorname{dog}($ TCS $=13)$, positive by IFAT but negative by Giemsa staining test, did not show any significant serum antibody reactivity towards leishmanial antigens. Another dog $(\mathrm{TCS}=7)$, positive by both tests, showed borderline Tot IgG and IgG2 ODs.

Within the HD group, sera from 36\% (26/71) of dogs were positive for Tot IgG which suggests an asymptomatic infection. All demonstrated IgG2 antibody reactivity towards leishmanial antigens and only three presented an IgG1 reactivity lower than the cut-off value (Fig. 1A). Similarly to the SD group, Tot IgG and IgG2 antibodies were more reactive than IgG1 antibodies in AD. Interestingly, among the remaining $45 \mathrm{HND}$ (negative for Tot IgG), 62\% (27/45) of dogs were negative for both IgG isotypes while $31 \%$ (14/45) and 6.6\% (3/45) of dogs showed, even though ODs were borderline, an exclusive IgG1 or IgG2 reactivity, respectively (Fig. 1A).

Comparison between $\mathrm{AD}$ and $\mathrm{SD}$ groups indicate, at both tested serum dilutions, lower levels of Tot IgG, IgG2 but also of IgG1 antibodies in AD (Fig.1A). Interestingly, the AD group displayed a slightly higher frequency of positive $\operatorname{IgG} 1$ reactivity comparatively to SD but only at 1:100 serum dilution ( $88 \%$ for $\mathrm{AD}$ versus $82 \%$ for $\mathrm{SD}$ ). The production of Tot IgG in dog's sera correlated positively with that of $\mathrm{IgG} 2$ in both $\mathrm{AD}$ (at dilution $1: 500, \mathrm{r}=0.83 ; P<0.0001$; Fig. 1B) and SD groups (at dilution 1:2,500, $\mathrm{r}=0.92, P<0.0001$; Fig. 1B) whereas no significant correlation was observed with the IgG1 isotype ODs.

Considering the controversial findings regarding the IgG isotype profile associated with cVL and the results obtained in the present study showing that neither the reactivity of the IgG1 isotype nor that of the IgG2 with leishmanial antigens is relatively higher in AD than in SD, we opted to re- 
analyze dogs' serum reactivity in order to determine their antibody titers. Serum samples from 12 $\mathrm{AD}$ and $16 \mathrm{SD}$, which presented high IgG1 and/or IgG2 reactivity were tested by ELISA at dilutions $100,400,1,600$ and 6,400. Obtained data clearly confirms that SD produce higher serum levels of IgG1 and IgG2 comparatively to AD (Fig. 1C).

Most of the investigations of the humoral response of L. infantum naturally-infected dogs have used either whole promastigote extracts or soluble leishmanial antigens obtained after promastigote lysis/sonication followed or not by removal of insoluble material. The use of such antigenic preparations which contain nuclear and cytoplasmic components, conserved between species, could have resulted in non specific binding of IgG1 and/or IgG2 dog's antibodies. In order to check whether the use of Leishmania-specific antigens would generate results that corroborate those obtained herein with total soluble antigens, we assessed the reactivity of $\operatorname{IgG}$ isotypes using recombinant LACK (Fig. 2A and B) and LeIF (Fig. 2C and D) proteins. Dogs from HND were tested in parallel. Results indicate that IgG1 (Fig. 2.A and C) and IgG2 (Fig. 2.B and D) productions by $\mathrm{AD}$ and SD infected dogs were significantly different when compared with HND $(P$ $<0.02$ ). For both recombinant proteins, the IgG1 subclass levels and especially those of IgG2, were significantly higher in SD compared to AD sera (at dilution 1:400, $P=0.0006$, data not shown), corroborating the data from native antigens-based ELISA. The frequency of positive reactions (Freq \%) was constantly higher for SD group, for both isotypes and both recombinant proteins (Fig. 2).

\subsection{Evaluation of Tot IgG, IgG1 and IgG2 autoantibodies reactivity of dog's sera by ELISA}

Considering that (i) the conflicting results reported in the literature were often related to the IgG1 isotype where authors have considered it as a surrogate marker of the asymptomatic stage (ii) the relatively higher frequency of IgG1 binding to leishmanial antigens observed in AD (88 and $82 \%$ for $\mathrm{AD}$ and $\mathrm{SD}$, respectively) despite a significant lower levels of specific IgG1, we hypothesized that their binding to leishmanial antigens could be non specific. Serum samples form 22 AD, 34 SD, and 27 HND were tested by HEp-2-ELISA at dilutions 1:100 and 1:200. Results 
indicate the presence of Tot $\mathrm{IgG}, \mathrm{IgG} 1$ and $\mathrm{IgG} 2$ autoantibody reactivity in the sera of $\mathrm{AD}$ and SD groups and at a lesser extent in HND (Fig. 3). The highest reactivity of Tot IgG and IgG2 was obtained for the SD group and all comparisons were statistically significant (Fig. 3). Moreover, we observed a significant difference at 1:100 dilution between the level of IgG1 autoantibodies in AD comparatively to HND $(P=0.017)$ but not to SD group. Interestingly, AD group demonstrated again an increased frequency of IgG1 antibodies binding to HEp-2 antigens (50\% versus $26 \%$ considering the whole group and $60 \%$ versus $28 \%$ considering only IgG1-positive dogs in $L$. infantum-ELISA).

Tot IgG reactivity with HEp-2 antigens correlated positively with that of IgG2, in both AD and SD groups (Fig. 3B) whereas, as seen for leishmanial antigens, no significant association was obtained using IgG1 ODs at any tested serum dilution. Moreover, L. infantum-IgG2 ODs correlated with HEp-2-IgG2 ODs in SD $(r=0.41 ; P=0.014)$ but not in AD $(r=0.35 ; P=0.1$; Fig. $3 C)$.

Considering the heterogeneity of dog's antibody reactivity, sera from 5 SD and 6 AD, which showed higher than $3 \mathrm{SD}$ of the mean of CTD group, were selected for more detailed analyses of their IgG1 and IgG2 subclass reactivity to HEp-2 antigens. Samples diluted at 1:100, 1:200, 1: 400 and 1:800 were tested by HEp-2 ELISA. Binding curves presented in Fig. 4 indicate a higher IgG1 reactivity against HEp-2 extracts in AD comparatively to SD (at 1:100 dilution, $P=0.031$ ) whereas the latter presented higher $\operatorname{IgG} 2$ reactivity (at 1:100 dilution $P=0.0033$ ).

Moreover, we observed that autoantibodies against human albumin (Fig. 5A), ds-DNA (Fig. 5 B) and human transferrin (Fig. 5 C) in dogs' sera were dominated in all analyzed groups by the $\operatorname{IgG} 2$ isotype. The reactivity of $\mathrm{IgG} 2$ autoantibodies was stronger in SD relatively to HND $(P<0.0001)$ and to $\mathrm{AD}(P<0.003)$. The reactivity of the $\mathrm{IgG} 1$ isotype with albumin and ds-DNA, even though statistically non-significant, was recorded at a higher frequency in $\mathrm{AD}$ and SD groups comparatively to HND. A significant increase of $\mathrm{IgG} 1$ and $\mathrm{IgG} 2$ anti-transferrin antibodies is recorded in $\mathrm{SD}$ as well as in $\mathrm{AD}$ in comparison with healthy dogs from endemic areas. The relative 
reactivity of $\operatorname{IgG} 2$, but not of $\mathrm{IgG1}$, anti-transferrin was significantly higher in SD comparatively to $\mathrm{AD}(P=0.0004)$.

In order to better characterize the specificity of IgG1 and IgG2 autoantibodies, we checked by competitive ELISA whether their binding to leishmanial antigens in L. infantum-ELISA experiments was specific. Two-fold serially diluted serum samples obtained from AD and SD were pre-incubated with a fixed amount of leishmanial antigens then added to HEp-2-coated plates. Serum dilutions without inhibitor were used as controls. Results show that, while the degrees of inhibition of $\operatorname{IgG} 2$ binding by the leishmanial antigens were similar between groups (at dilution $1: 150 ; 49 \%$ of inhibition in $\mathrm{AD}$ and $48 \%$ in $\mathrm{SD})$, those of the IgG1 were more important in SD (70\% and $30 \%$ for $\mathrm{SD}$ and $\mathrm{AD}$, respectively), suggesting that the proportion of cross-reactive IgG1/non cross-reactive autoantibodies in more important in SD than in AD (Fig. 6 A). The addition of higher amounts of leishmanial antigens did not result in complete (100\%) inhibition of antibody binding to HEp-2 coated plates. Moreover, the lower percentage of IgG2 inhibition comparatively to IgG1 (48\% versus 70\%) in SD (Fig. 6 B) is in favor that the relative proportion of cross-reactive IgG1 autoantibodies is higher than that of their IgG2 counterpart. In the opposite, the proportion of cross-reactive $\operatorname{IgG} 2$ autoantibodies in AD is more important than its counterpart IgG1.

\subsection{Western blot analysis}

The reactivity of $\mathrm{AD}$ and $\mathrm{SD}$ serum samples towards HEp-2 antigens was also compared by WB to check whether dog's autoantibodies have targeted or not the same proteins. Examples of IgG1 (lanes a) and IgG2 (lanes b) reactivity, illustrated in Fig. 7, showed that both groups produced highly heterogeneous immunodetection patterns. However, it is noteworthy that IgG1 antibodies from six out of ten $\mathrm{AD}$ seem to be more reactive than $\mathrm{IgG} 2$ antibodies (examples AD1, AD4 and AD7) whereas the opposite was observed for SD serum samples. IgG1 and IgG2 autoantibodies from late stage SD (high TCS, SD9 to SD12) seem also more reactive with HEp-2 proteins. IgG1 and $\mathrm{IgG} 2$ reactivity was mostly targeting the proteins with $72,62,46,43,35,30,23$, and $16 \mathrm{kDa}$ of 
MW. The protein band with $16 \mathrm{kDa}$ was more frequently reactive in AD group. Henriksson et al., (1998) analyzed by HeLa cell nuclear extract immunoblots the serum reactivity of ANA positive dogs and showed that a $43 \mathrm{kDa}$ nuclear protein, previously described as hnRNP G, was the most frequently recognized autoantigen.

\section{Discussion}

We aimed the present study to analyze the specific and autoimmune repertoires of $L$. infantum-infected dogs for a better understanding of the parasite-induced development of the specific and non specific humoral immune responses. Indeed, many investigations of the specific antibody response have led to inconsistent results where (i) authors claimed that either IgG1 or IgG2 is the isotype marker of the asymptomatic infection status whereas (ii) others suggested that the specific antibody responses cannot be used as surrogate markers of the $\mathrm{T}$ cell immune responses or as an indication of the clinical status of infected dogs (Quinnel et al., 2003; Strauss-Ayali et al., 2007), highlighting the difficulties to establish a typical immunological response profile of $L$. infantum-infected dogs.

The results presented herein, which analyzes for the first time the IgG1-IgG2 humoral responses of L. infantum-infected domestic dogs living in Tunisian endemic areas, oppose the studies reporting higher levels of specific IgG1 or IgG2 during the subclinical phase of dog's infection but also those describing that $\mathrm{AD}$ and SD produce similar levels of specific IgG2 (de Freitas et al., 2012; Lima et al., 2017). Results obtained by ELISA based on native soluble $L$. infantum antigens showed that the levels of specific Tot IgG, IgG1 and IgG2 were constantly higher during the active clinical infection. The use of leishmanial LACK and LeIF recombinant proteins corroborated the obtained results despite a lower assay's sensitivity (except for LeIF-IgG2). An overall strong degree of correlation was observed between Tot $\operatorname{IgG}$ and $\operatorname{IgG} 2$ ODs but not with IgG1 ODs, in SD and AD groups. Moreover, we did not observe, as described by Quinnell et al. (2003), that the titers of specific IgG1 exceed those of IgG2 neither in SD nor in AD. Individual 
specific IgG1 levels were constantly lower than those of IgG2 in all dogs (Almeida et al., 2005), except in one AD, two SD and interestingly in seven HND. The presence in HND of an exclusive $\operatorname{IgG} 1(31 \%$, median $\mathrm{OD}=0.4)$ or $\operatorname{IgG} 2(6.6 \%$, median $\mathrm{OD}=0.8)$ reactivity with leishmanial antigens, raises the possibility that, despite Tot IgG ODs lower than cut-off value, dogs may already have been infected with $L$. infantum and that blood sampling occurred at a time period where their immune status precedes that of AD. If our hypothesis was true, it would suggest that healthy dogs with undetectable Tot IgG but with positive IgG1 and/or IgG2 ODs, may be included in the AD group.

Several hypotheses were previously proposed to explain such inconsistencies that raised despite the use of the same Leishmania strain, the investigation of the same endemic areas and the use of very similar experimental ELISA protocols based on commercial polyclonal or monoclonal anti-dog antibodies produced from the same companies. Hypotheses were more often related to the use of polyclonal instead of monoclonal anti-IgG isotypes, considered less sensitive, rather than to differences in the degree of specificity of the Leishmania-antigenic preparations caused by the cross-reactivity with other environmental pathogenic antigens. In addition, data variations resulting from (i) technical differences among which the choice of the reference population and of the cut-off value (Greiner et al., 2000; de Arruda et al., 2013), and from (ii) biological differences such as blood sampling period which could lead to differences in dog's immune status, were also discussed in the literature.

It is noteworthy that the molecular mimicry between Leishmania and host antigens suspected in the development of autoimmunity, could also be responsible for the "non-specific binding" of human's or dogs' sera, affecting hence the specificity of the serology assays. Indeed, Argov et al. (1989) detected the presence of IgG autoantibodies against Sm, RNP and SS-B nuclear antigens in the sera of 83,86 , and $73 \%$ of $\mathrm{hVL}$ cases, respectively but in less than $25 \%$ of cutaneous leishmaniasis patients. Authors showed by competitive ELISA that the binding of human VL serum antibodies to RNP could be inhibited after prior incubation of serum with leishmanial membrane 
antigens, implying that, besides the polyclonal activation of $\mathrm{B}$ lymphocytes, the production of autoimmune antibodies might have resulted from antigen cross-reactivity between leishmanial antigens and RNP. In addition, Louzir et al. (1994) reported an increase of polyreactive anti-tubulin, anti-actin and anti-myosin $\operatorname{IgG}$ natural autoantibodies in 86, 68 and $57 \%$ of Tunisian children with VL, respectively.

The production of circulating immune complexes (Lopez et al., 1996) and autoantibodies such as antinuclear antibodies (anti-DNA, RNA, nuclear proteins and their molecular complexes), and anti-mammalian basal membrane glycoproteins and cerebrosides, and anti-Histone, was previously described in L. infantum-infected dog's sera (Pateraki et al., 1983; Lucena and Ginel, 1998; Bell et al., 1997; Smith et al., 2004). Ferrer (1992) and Lucena et al. (1996) reported a 30 and $15.9 \%$ incidence of antinuclear antibodies in dogs with natural Leishmania infection, respectively. Pateraki et al. (1983) demonstrated by ELISA that $250(95 \%)$ and 248 (94\%) dogs out of 260 with cVL, all positive for $L$. donovani antigens, have anti-actin and anti-tubulin IgG autoantibodies, respectively. Ginel et al. (2008) reported that $88 \%$ of infected dogs with glomerulonephritis had high anti-histone antibodies. However, to our knowledge, no study analyzed the profile of IgG1 or IgG2 autoantibodies during the subclinical or the acute phase of dog's infection with L. infantum. Our results from ELISA-HEp-2 indicate the presence of an autoantibody activity in all groups including HND. Indeed, for the latter, we observed as described by Argov et al. (1989), investigating human VL, a relatively higher reactivity of endemic controls comparatively to the negative control group. This suggests that, if in case these dogs were previously infected, the polyclonal activation of B cells producers of natural antibodies occurred during the early stages of dog's infection.

Moreover, we observed that the reactivity of Tot $\mathrm{IgG}$ and $\mathrm{IgG} 2$ with nuclear and soluble HEp-2 extracts was significantly higher in SD, in terms of ODs and positive reactivity (\%). Thus, $100 \%$ and $74 \%$ of tested SD presented Tot IgG and IgG2 serum binding to HEp-2 whereas only $43 \%$ and $68 \%$ of $\mathrm{AD}$ were positive. Conversely, seroprevalence of IgG1 subclass was higher in AD 
(50\%) than in SD (26\%). Positive binding to HEp-2 proteins for SD group was independent of parasitological tests' results and was present in positive and negative serum samples. It was also present in AD serum samples reactive with L. infantum extracts, LACK and LeIF proteins, implying that the increase of autoantibody levels in these dogs was associated to Leishmania infection. In addition, we show that for the IgG1 isotype, the autoantibody response in AD was mainly directed against antigenic determinants that do not cross-react with Leishmania parasite (only $30 \%$ of inhibition by leishmanial antigens) whereas it was dominated by cross-reactive IgG1 antibodies in sick dogs (70\% of inhibition by leishmanial antigens). For the IgG2 isotype, and for both dogs' groups cross-reactive and non cross-reactive autoantibodies seem to be similarly represented.

Furthermore, it has been previously reported that the development of autoimmune reactions in human and canine VL could contribute to multiple alterations among which normocytic anemia, renal, hepatic and vascular alterations. Thus, the increase of the concentration of circulating immune complexes and their deposition in kidney tissue could be responsible for necrosis and impairment of the renal function, one of the leading causes of death in cVL (Brandonisio et al., 1990; Ciaramella, P. and Corona, M., 2003; Baneth et al., 2008; Koutinas and Koutinas, 2014). Transferrin is a plasma protein that transports iron through the blood to the liver, spleen and bone marrow and low serum transferrin levels was previously associated with anemia and chronic liver disease. Toet et al. (2014) found that Sarcoptes scabiei infested pigs produce antibodies to albumin and to iron-binding proteins including ferritin and transferrin. The development of natural $\operatorname{IgG}$ autoantibodies against transferrin in Leishmania-infected dogs, particularly in SD, could be a possible mechanism that lowers protein and iron levels, decreases hemoglobin production (due to lack of iron), leading to anemia and impairment of liver function. Further investigations, such as the measure of iron, hemoglobin and transferrin concentrations in dogs' infected sera, are however required to check whether these autoantibodies may trigger the development of anemia or liver disease, characteristic features of cVL. 


\section{Conclusion}

In this paper, ELISA results based on L. infantum native and recombinant proteins indicate that in a cohort of domestic dogs living in Tunisian endemic areas, the antibody titers of specific Tot IgG, IgG1 and IgG2 were constantly more elevated during the active phase of infection and that IgG2 is the predominant subclass present in infected AD and SD. The increase of autoantibodies' production starts since the subclinical stages and is characterized by relatively low levels of crossreactive $\operatorname{IgG} 1$ antibodies. Conversely, $\mathrm{IgG} 1$ cross-reactive antibodies dominate the autoantibody repertoire of SD. Taken together, our findings are in favor that the discordance described in previous studies could result from low specificity caused by the interference of a variety of autoantibodies, including natural and cross-reactive antibodies.

\section{Author Contributions}

AS conceived the paper, designed and performed the ELISA and western blotting experiments, analyzed the data and wrote the paper. AC participated in drafting and writing the manuscript. RBE veterinary physician collected healthy dogs' blood samples and critically revised the paper. MM veterinary physician established clinical dog's diagnosis, collected blood samples and revised the paper. MG participated in manuscript's revision. All authors approved the final manuscript version.

\section{Declaration of interest: none}

\section{Acknowledgements}

The authors acknowledge the dogs' owners for their contribution to the study. This research did not receive any specific grant from funding agencies in the public, commercial, or not-for-profit sectors. 


\section{References}

Abbehusen, M.M.C., Almeida, V.D.A., Solcà, M.D.S., Pereira, L.D.S., Costa, D.J., Gil-Santana, L., Bozza, P.T., Fraga, D.B.M., Veras, P.S.T., Dos-Santos, W.L.C., Andrade, B.B., Brodskyn, C.I., 2017. Clinical and immunopathological findings during long term follow-up in Leishmania infantum experimentally infected dogs. Sci. Rep. 7, 15914.

Almeida, M.A.O., Jesus, E.E.V., Sousa-Atta, M.L.B., Alves, L.C., Berne, M.E.A. Atta, A.M., 2005. Antileishmanial antibody profile in dogs naturally infected with Leishmania chagasi. Vet. Immunol. Immunopathol. 106, 151-158.

Alvar, J., Vélez, I.D., Bern, C., Herrero, M., Desjeux, P., Cano, J., Jannin, J., den Boer, M., WHO Leishmaniasis Control Team, 2012. Leishmaniasis worldwide and global estimates of its incidence. PLoS ONE. 7, e35671.

Anam, K., Afrin, F., Banerjee, D., Pramanik, N., Guha, S.K., Goswami, R.P., Gupta, P.N., Saha, S.K., Ali, N., 1999. Immunoglobulin subclass distribution and diagnostic value of Leishmania donovani antigen-specific immunoglobulin G3 in Indian kala-azar patients. Clin. Diagn. Lab. Immunol. 6, 231-235.

Argov, S., Jaffe, C.L., Krupp, M., Slor, H., Shoenfeld, Y., 1989. Autoantibody production by patients infected with Leishmania. Clin. Exp. Immunol., 76, 190-197.

Baneth, G., Koutinas, A. F., Solano-Gallego, L., Bourdeau, P. And Ferrer, L., 2008. Canine leishmaniosis - new concepts and insights on an expanding zoonosis: part one. Trends in Parasitology 24, 324-330.

Bettini, S., Gradoni, L., 1986. Canine leishmaniasis in the Mediterranean area and its implications for human leishmaniasis. Insect. Sci. Appl. 7, 241-245.

Bhattacharyya, T., Ayandeh, A., Falconar, A.K., Sundar, S., El-Safi, S., Gripenberg, M.A., Bowes, D.E., Thunissen, C., Singh, O.P., Kumar, R., Ahmed, O., Eisa O., Saad, A., Silva Pereira, S., Boelaert, M., Mertens, P., Miles, M.A., 2014. IgG1 as a potential biomarker of post- 
chemotherapeutic relapse in visceral leishmaniasis, and adaptation to a rapid diagnostic test. PLoS Negl Trop Dis. 8, e3273.

Binder, S.R., Genovese, M.C., Merrill, J.T., Morris, R.I., Metzger, A.L., 2005. Computer-assisted pattern recognition of autoantibody results. Clin. Diagn. Lab. Immunol. 12, 1353-1357.

Boceta, C., Alonso, C., Jimenez-Ruiz, A., 2000. Leucine rich repeats are the main epitopes in Leishmania infantum PSA during canine and human visceral leishmaniasis. Parasite Immunol. $22,55-62$.

Böhme, M.W., Evans, D.A., Miles, M.A. Holborow, E.J., 1986. Occurrence of autoantibodies to intermediate filament proteins in human visceral leishmaniasis and their induction by experimental polyclonal B-cell activation. Immunology. 59, 583-588.

Bourdoiseau, G., Bonnefont, C., Hoareau, E., Boehringer, C., Stolle, T., Chabanne, L., 1997. Specific IgG1 and IgG2 antibody and lymphocyte subset levels in naturally Leishmania infantum infected treated and untreated dogs. Vet. Immunol. Immunopathol. 59, 21-30.

Brandonisio, O., Carelli, G., Altamura, M., Varvara, B., Ceci, L., 1990. Circulating immune complexes and autoantibodies in canine leishmaniasis. Parassitologia. 32, 275-281.

Cabral, M., O’grady, J.E., Gomes, S., Sousa, J.C., Thompson, H., Alexander, J., 1998. The immunology of canine leishmaniasis: strong evidence for a developing disease spectrum from asymptomatic dogs. Vet. Immunol. Immunopathol. 76, 173-180.

Cardoso, L., Schallig, H.D.F.H., Cordeiro-da-Silva, A., Cabral, M., Alunda, J.M., Rodrigues, M., 2007. Anti-Leishmania humoral and cellular immune responses in naturally infected symptomatic and asymptomatic dogs. Vet. Immunol. Immunopathol. 117, 35-41.

Carson, C., Quinnell, R.J., Day, M.J., Courtenay, O., 2010. Comparison of monoclonal and polyclonal antibodies for the detection of canine $\operatorname{IgG1}$ and $\operatorname{IgG} 2$, and associations with infection outcome in Leishmania infantum naturally infected dogs. Vet Immunol Immunopathol. 133, 264-268. 
Carvalho, E.M., Andrews, B.S., Martinelli, R., Dutra, M., Rocha, H., 1983. Circulating immune complexes and rheumatoid factor in schistosomiasis and visceral leishmaniasis. Am. J. Trop. Med. Hyg. 32, 61-68.

Ciaramella, P., Corona, M., 2003. Canine leishmaniasis: clinical and diagnostic aspects. VetLearn $25,358-368$.

de Freitas, J.C., Lopes-Neto, B.E., de Abreu, C.R., Coura-Vital, W., Braga, S.L., Reis, A.B., 2012. Nunes-Pinheiro DC Profile of anti-Leishmania antibodies related to clinical picture in canine visceral leishmaniasis. Res. Vet. Sci. 93, 705-709.

Deplazes, P., Smith, N.C., Arnold, P., Lutz, H., Ecker, J., 1995. Specific IgG1 and IgG2 antibody response of dogs to Leishmania infantum and other parasites. Parasite Immunol. 17, 451-458.

Ferrer, L.M., 1999. Clinical aspects of canine leishmaniasis. In: Canine leishmaniasis: an update. Barcelona, Spain. Wiesbaden: Hoechst Roussel Vet, 6-9.

Galvão-Castro, B., Sá Ferreira, J.A., Marzochi, K.F., Marzochi, M.C., Coutinho, S.G., Lambert, P.H., 1984. Polyclonal B cell activation, circulating immune complexes and autoimmunity in human american visceral leishmaniasis. Clin. Exp. Immunol. 56, 58-66.

Ginel, P.J., Camacho, S., Lucena, R., 2008. Anti-histone antibodies in dogs with leishmaniasis and glomerulonephritis. Res Vet Sci. 85, 510-514.

Greiner, M., Gardner, I.A., 2000. Epidemiologic issues in the validation of veterinary diagnostic tests. Prev. Vet. Med. 45, 3-22.

Grennwood, B.M., 1974. Possible role of a B-cell mitogen in hypergammaglobulinemia in malaria and trypanosomiasis. The Lancet. 303, 435-436.

Henriksson, E.W., Hansson, H., Karlsson-Parra, A., Pettersson, I., 1998. Autoantibody profiles in canine ANA-positive sera investigated by immunoblot and ELISA. Vet. Immunol. Immunopathol. 61, 157-170.

Iniesta, L., Gállego, M., Portús, M., 2005. Immunoglobulin G and E responses in various stages of canine leishmaniasis. Vet. Immunol. Immunopathol. 103, 77-81. 
Jamal, F., Shivam, P., Kumari, S., Singh, M.K., Sardar, A.H., Pushpanjali, Murugesan, S., Narayan, S., Gupta, A.K, Pandey, K., Das, V.N.R, Ali, V., Bimal, S, Das, P., Singh, S.K., 2017. Identification of Leishmania donovani antigen in circulating immune complexes of visceral leishmaniasis subjects for diagnosis. PLoS One. 12, e0182474.

Koutinas, A.F., Koutinas, C.K., 2014. Pathologic mechanisms underlying the clinical findings in canine leishmaniasis due to Leishmania infantum/chagasi. Vet. Pathol. 51, 527-38.

Liberopoulos, E., Pappas, G., Kostoula, A., Drosos, A., Tsianos, E., Elisaf, M., 2003. Spectrum of autoimmunity and dysproteinemia in patients with visceral leishmaniasis. Clin. Microbiol. Infect. 9, 417.

Lima, L.V.D.R., Carneiro, L.A., Campos, M.B., Vasconcelos Dos Santos, T., Ramos, P.K., Laurenti, M.D., Teixeira, C.E.C, Silveira, F.T., 2017. Further evidence associating IgG1, but not $\operatorname{IgG} 2$, with susceptibility to canine visceral leishmaniasis caused by Leishmania $(L$. infantum chagasi-infection. Parasite. 24, 37.

Lopez, R., Lucena, R., Novales, M., et al., 1996. Circulating immune complexes and renal function in canine leishmaniasis. Zentralbl Veterinarmed B.43, 469-474.

Louzir, H., Belal-Kacemi, L., Sassi, A., Laouini, D., Ben Ismail, R., Dellagi, K., 1994. Natural autoantibodies, IgG antibodies to tetanus toxoid and CD5+ B cells in patients with Mediterranean visceral leishmaniasis. The Leishmania Study Group. Clin. Exp. Immunol. 95, 479-484.

Lucena, R., Ginel, P.J., Lopez, R., Novales, M., Martin, E., Molleda, J.M., 1996. Antinuclear Antibodies in Dogs with Leishmaniasis. J. Vet. Med. Ser. A 43, 255-259.

Lucena, R. and Ginel, P. J. (1998). Immunoglobulin isotype distribution of antinuclear antibodies in dogs with leishmaniasis. Res. Vet. Sci. 65, 205-207.

Montes, C.L., Acosta-Rodríguez, E.V., Merino, M.C., Bermejo, D.A., Gruppi, A., 2007. Polyclonal B cell activation in infections: infectious agents' devilry or defense mechanism of the host? $\mathrm{J}$ Leukoc Biol. 82, 1027-1032. 
Nieto, C.G., Garcia-Alonso, M., Requena, J.M., Mirón, C., Soto, M., Alonso, C., Navarrete, I., 1999. Analysis of the immune response against total and recombinant antigens of Leishmania infantum: correlation with disease progression in canine experimental leishmaniasis. Vet. Immunol. Immunopathol. 67, 117-130.

Pateraki, E., Portocala, R., Labrousse, H., Guesdon, J.L., 1983. Antiactin and antitubulin antibodies in canine visceral leishmaniasis. Infect. Immun. 42, 496-500.

Pinelli, E., Ellick-Kendrick, R., Wagenaar, J., Bernadina, W., Real, G., Ruitenberg, J., 1994. Cellular and humoral immune responses in dogs experimentally and naturally infected with Leishmania infantum. Infect. Immun. 62, 229-235.

Quinnell, R.J., Courtenay, O., Garcez, L.M., Kaye, P.M., Shaw, M.A., Dye, C., Day, M.J., 2003. IgG subclass responses in a longitudinal study of canine visceral leishmaniasis. Vet Immunol Immunopathol. 91, 161-168.

Reis, A.B., Teixeira-Carvalho, A., Vale, A.M., Marques, M.J., Giunchetti, R.C., Mayrink, W., Guerra, L.L., Andrade, R.A., Corrêa-Oliveira, R., Martins-Filho, O.A., 2006. Isotype patterns of immunoglobulins: Hallmarks for clinical status and tissue parasite density in Brazilian dogs naturally infected by Leishmania (Leishmania) chagasi. Vet. Immunol. Immunopathol. 112, $1012-1116$.

Sassi, A., Kaak, O., Benammar Elgaaied, A., 2015. Identification of immunodominant Leishmania major antigenic markers of the early C57BL/6 and BALB/c mice infection stages. Parasite Immunol. 37, 544-552.

Smith, B. E., Tompkins, M. B. and Breitschwerdt, E. B., 2004. Antinuclear antibodies can be detected in dog sera reactive to Bartonella vinsonii subsp. berkhoffii, Ehrlichia canis, or Leishmania infantum antigens. J. Vet. Inter. Med. 18, 47-51.

Strauss-Ayali, D., Baneth, G., Jaffe, C.L., 2007. Splenic immune responses during canine visceral leishmaniasis. Vet. Res. 38, 547-564. 
Teixeira Neto, R.G., Giunchetti, R.C., Carneiro, C.M., Vitor, R.W., Coura-Vital, W., Quaresma, P.F., Ker, H.G., de Melo, L.A., Gontijo, C.M., Reis, A.B., 2010. Relationship of Leishmaniaspecific IgG levels and IgG avidity with parasite density and clinical signs in canine leishmaniasis. Vet. Parasitol. 169, 248-57.

Toet, H.M., Fischer, K., Mounsey, K.E., Sandeman, R.M., 2014. Autoantibodies to iron-binding proteins in pigs infested with Sarcoptes scabiei. Vet. Parasitol. 205, 263-270.

Tunccan, O.G., Tufan, A., Telli, G., Akyürek, N., Pamukçuoğlu, M., Yılmaz, G., Hızel, K., 2012. Visceral Leishmaniasis Mimicking Autoimmune Hepatitis, Primary Biliary Cirrhosis, and Systemic Lupus Erythematosus Overlap. Korean J. Parasitol. 50, 133-136. 


\section{Figure legends}

Figure 1. ELISA results of dog's serum samples reactivity with leishmanial antigens. (A) Comparison of the IgG, IgG1 and IgG2 level's between HND ( $\mathrm{n}=45), \mathrm{AD}(\mathrm{n}=26)$ and SD $(\mathrm{n}=34)$. Each dot represents one individual dog's serum tested at dilutions 1:100 and 1:500. Horizontal lines represent the median value of each plot. The cut-off values for dilution 1:100 (dashed lines) were determined from $26 \mathrm{CTD}$ (mean $+3 \mathrm{SD}$ ) and used to check for positive serum reactivity. The seroprevalence $(\%)$ of positive dogs is indicated for the dilution 1:100. The unpaired $t$-test of Student's was used to calculate significance levels between the OD values; $P$ values from comparison between AD and SD groups are shown. Significant differences between non infected (HND) and infected dogs (AD or SD) were obtained for all antibody levels $(P<0.0001)$. (B) Correlations between the OD values of Tot IgG and IgG2 in SD (dilution 1:2500) and AD (dilution 1:500) groups. The Spearman's coefficient of correlation $(\mathrm{r})$ and the $P$ values are indicated on the graph. (C) Symptomatic L. infantum-infected dogs produce higher levels of specific IgG1 and IgG2 antibodies than AD against leishmanial antigens. Sera collected from 12 AD, 16 SD and 5 CTD were diluted at 1:100, 1:400, 1:1600 and 1:6400 and tested by ELISA for their reactivity towards leishmanial antigens. Results are expressed as mean of OD \pm 1 standard error of the mean (SEM). 

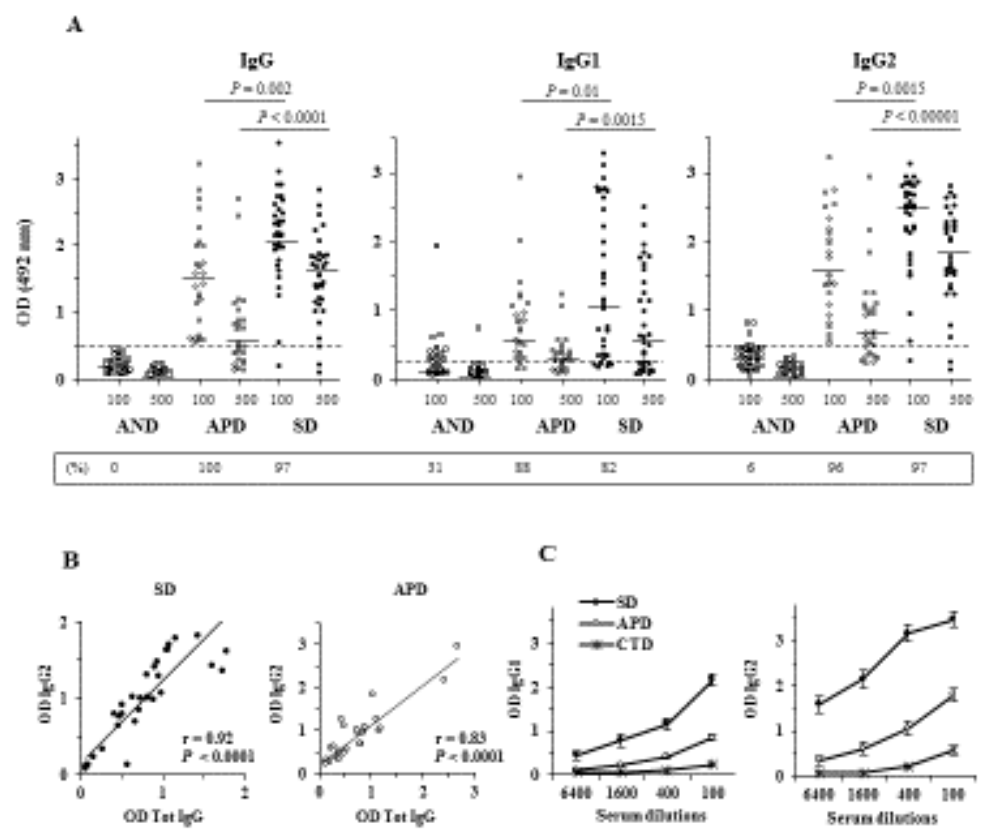

Figure 2. Reactivity of IgG1 and IgG2 antibodies against LACK and LeIF recombinant proteins. Sera from $12 \mathrm{HND}, 20 \mathrm{AD}$ and $15 \mathrm{SD}$ were diluted at 1:100 and incubated on LACK (A, B) or LeIF-coated plates (C, D). Values in box plots reflect the median and the interquartile range of IgG1 (A, C) and IgG2 (B, D) serum reactivity. The boxes represent $95 \%$ of the values. Cut-off values were determined from 6 CTD dogs (mean +2 SD). $P$ values from comparison by the unpaired Student's t-test between AD and SD are shown. All comparisons between HND and AD or SD serum sample's reactivity were statistically significant $(P<0.04$ with $\mathrm{AD}$, and $P<0.001$ with SD). 


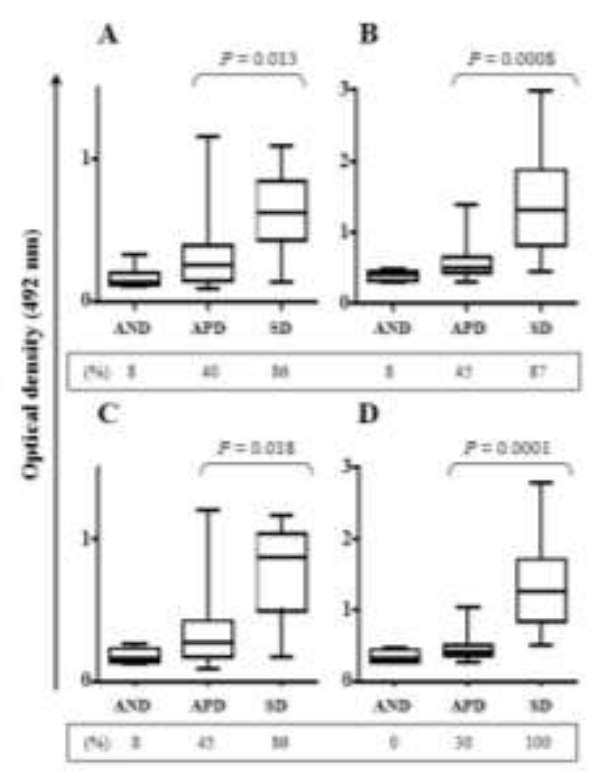

Figure 3. ELISA of IgG, IgG1 and IgG2 reactivity of dog's sera to HEp-2 cell extracts. In panel A, the reactivity of $\operatorname{IgG}, \operatorname{IgG} 1$ and $\operatorname{IgG} 2$ antibodies was compared between HND ( $\mathrm{n}=27), \mathrm{AD}(\mathrm{n}=22)$ and SD (n=34). Horizontal lines represent the plots' median values. The cut-off values (dashed lines for dilution 1:100) were determined from $22 \mathrm{CTD}$ (mean $+3 \mathrm{SD}$ ) and used to check for serum positive reactivity. The seroprevalence $(\%)$ is indicated for the dilution 1:100. The unpaired $t$-test of Student's was used to calculate significance levels between OD values; $P$ values from comparison between HND, AD and SD groups are shown. Panel B shows the correlations between the OD values of Tot IgG and IgG2 in SD and AD groups. Panel C shows the correlations between IgG2 reactivity with HEp-2 and $L$. infantum antigens. The Pearson's r coefficients and the $P$ values are indicated on the graph. 

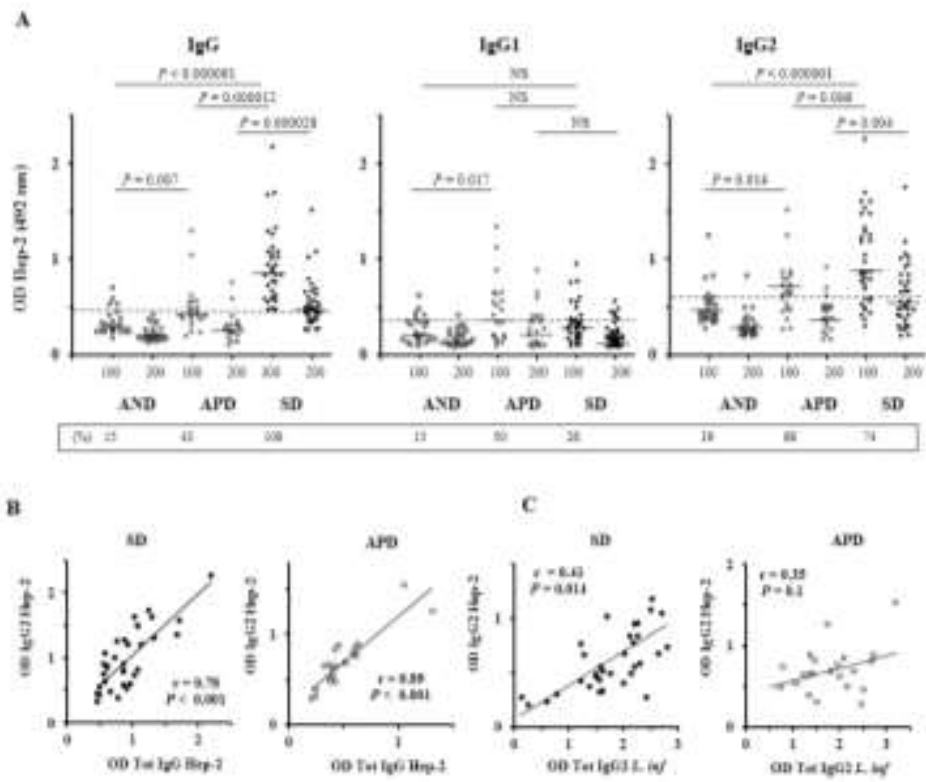

Figure 4. Asymptomatic $L$. infantum-infected dogs show higher reactivity of $\operatorname{IgG} 1$ antibodies against HEp-2 extracts than symptomatic dogs $(P<0.04)(\mathrm{A})$ whereas the latter present higher levels of IgG2 autoantibodies $(P<0.003)(B)$. Sera collected from 6 AD, 9 SD and 5 CTD were diluted at 1:100, 1:200, 1:400 and 1:800 and tested by ELISA. Results are expressed as mean of OD \pm 1 SEM.

A

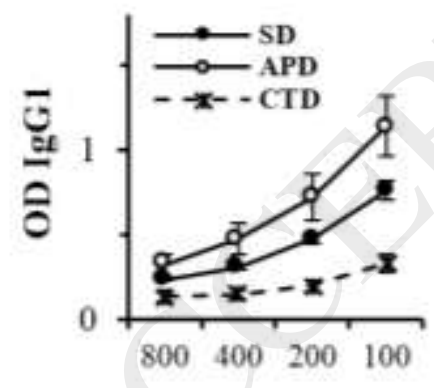

Serum dilutions
B

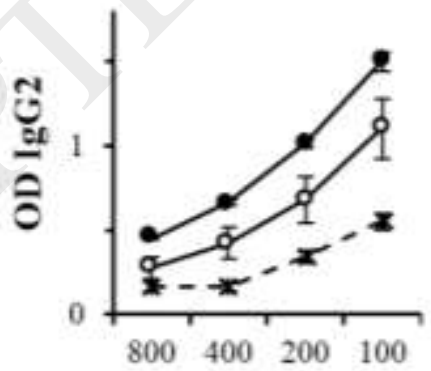

Serum dilutions

Figure 5. IgG1 and IgG2 reactivity to albumin (A), ds-DNA (B) and transferrin (C). Dogs' sera were from $\mathrm{HND}(\mathrm{n}=5$ to 16$), \mathrm{AD}(\mathrm{n}=10$ to 21$)$, and $\mathrm{SD}(\mathrm{n}=10$ to 32$)$. Serum samples from CTD ( $\mathrm{n}=5$ to 11$)$ were used to establish cut-off values for positivity at a dilution of 1:100 (dashed lines). 
Values given are the median $\mathrm{OD}_{492}$ of each group of dogs (horizontal lines). The Mann-Whitney U test was used for comparisons between groups. NS indicates non significant difference between groups. NS*: Comparison between only positive dogs was significant $(P<0.05)$.

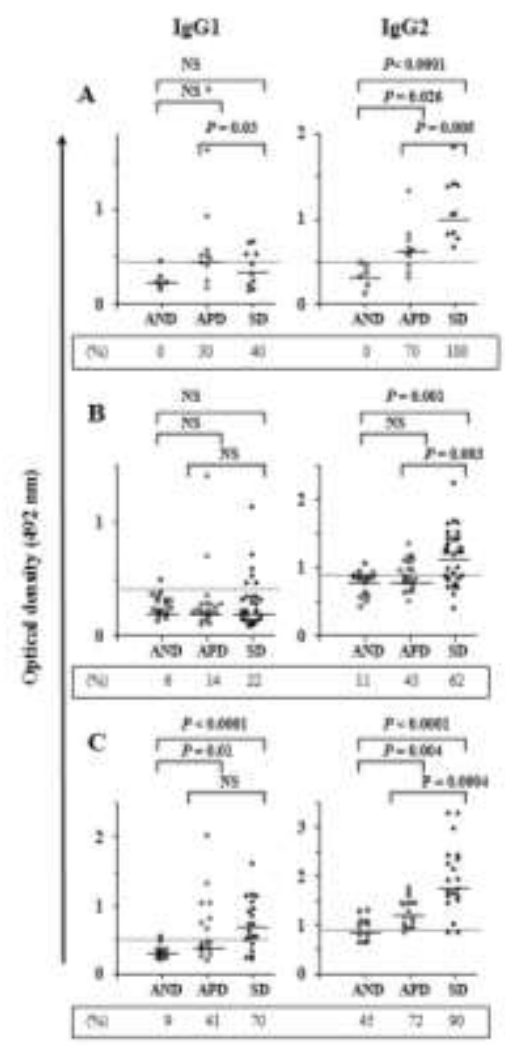

Figure 6. Competitive ELISA with HEp-2 coated plates. Dog's serum samples obtained from 8 AD (A) and 7 SD (B) which displayed anti-HEp-2 activity were incubated with (W) or without (WO) $100 \mu \mathrm{g} / \mathrm{ml}$ of leishmanial antigens. Sera were tested at two-fold serial dilutions from 1:150 to 1:1200. Results are expressed as mean \pm 1 SEM of OD. The percentage of inhibition of IgG1 and IgG2 binding to HEp-2 coated wells by leishmanial antigens is provided for 1:150 serum dilution. Decrease in antibody binding to HEp-2 antigens was significant by paired Student's $t$-test for both groups and isotypes. $P$ values are indicated in the figure for dilution 1:500. 


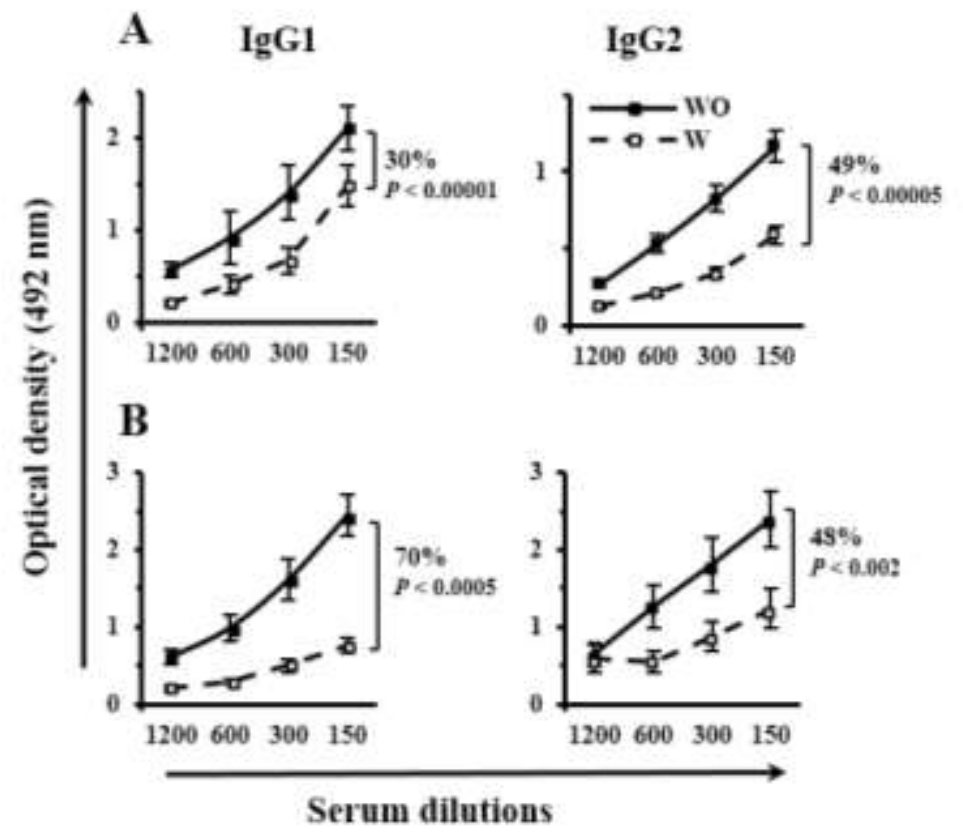

Figure 7. Immunodetection profiles of IgG1 (lanes a) and IgG2 (lanes b) antibody reactivity with HEp-2 proteins. Serum samples from 12 SD (panel A), 11 AD and 1 CTD (panel B) were diluted at 1:500 and tested against HEp-2 proteins after separation by electrophoresis on 15\% SDSacrylamide gels and blotting to nitrocellulose membranes. The TCS of SD samples is shown. Numbers indicate proteins' MWs. Serum samples from AD (AD1, AD4, AD5, AD7, and AD10) and from SD (SD7 and SD11) exhibited higher IgG1 reactivity than IgG2. 
A

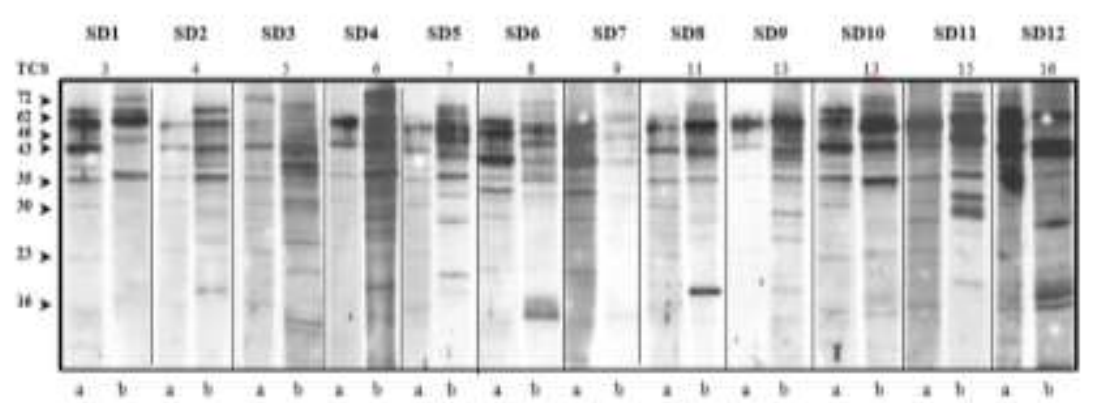

B

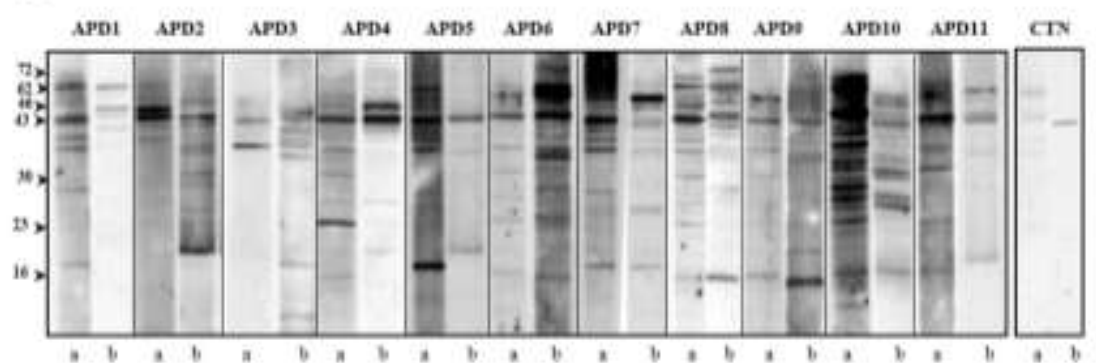

